\title{
O Programa de Educação pelo Trabalho para a Saúde (PET- Saúde) e a pandemia de Covid-19: ações possíveis para o apoio a pessoas em situação de rua e para o fortalecimento do controle social
}

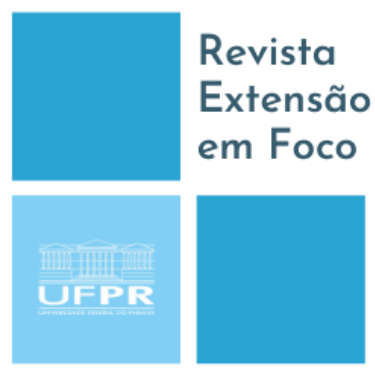

ISSN $2358-7180$

\author{
The Education through Work for Health Program \\ (PET-Saúde) and the Covid-19 pandemic: \\ possible actions to support homeless people and \\ to strengthen social control
}

\begin{abstract}
Luís Felipe Ferro ${ }^{1}$, Daiana Kloh Khalaf ${ }^{2}$, Rafael Gomes Ditterich ${ }^{3}$, Regina Maria Ferreira Lang ${ }^{4}$, Amanda Khetleen Gusso $^{5}$, Flávia Castro Rosa ${ }^{6}$, Isabela Cristina Santos Freire de Paula ${ }^{7}$, Karoline Fernanda Zamboni Bonan ${ }^{8}$, José Nilson de Freitas Ferreira ${ }^{9}$, Bruna Menezes Mincov ${ }^{10}$
\end{abstract}

\footnotetext{
${ }^{1}$ Doutor em Ciências e Pós-Doutor pelo Departamento de Psicologia Social e do Trabalho do Instituto de Psicologia da USP. Docente do Departamento de Terapia Ocupacional da Universidade Federal do Paraná (UFPR). Curitiba, Paraná, Brasil. E-mail: luisfelipeferro@ gmail.com. Orcid: https://orcid.org/0000-00018935-104X.

${ }^{2}$ Doutora em Enfermagem.Docente do Departamento de Enfermagem da Universidade Federal do Paraná (UFPR). Curitiba, Paraná, Brasil. E-mail: daianakloh@ufpr.br. Orcid: https://orcid.org/0000-0001-5770$\underline{7523}$.

${ }^{3}$ Doutor em Odontologia (Saúde Coletiva). Docente do Departamento de Saúde Coletiva da Universidade Federal do Paraná (UFPR). Curitiba, Paraná, Brasil. E-mail: prof.rafaelgd@gmail.com. Orcid: http://orcid.org/0000-0001-8940-1836.

${ }^{4}$ Mestra em Ciências da Saúde. Docente do Departamento de Nutrição da Universidade Federal do Paraná (UFPR), Curitiba, Paraná, Brasil. E-mail: reginalang@ufpr.br. Orcid: http://orcid.org/0000-0003-3613$\underline{1275}$.

${ }^{5}$ Discente de Enfermagem. Universidade Federal do Paraná (UFPR), Curitiba, Paraná, Brasil. E-mail: amandakgusso@gmail.com. Orcid: https://orcid.org/0000-0002-9887-9898.

${ }^{6}$ Discente de Odontologia. Universidade Federal do Paraná (UFPR), Curitiba, Paraná, Brasil. E-mail: fla.schiavinato@gmail.com. Orcid: https://orcid.org/0000-0002-3632-9911.

7 Discente de Odontologia. Universidade Federal do Paraná (UFPR), Curitiba, Paraná, Brasil. E-mail: isabelafreirep@gmail.com. Orcid: https://orcid.org/0000-0001-6996-0673.

8 Discente de Odontologia. Universidade Federal do Paraná (UFPR), Curitiba, Paraná, Brasil. E-mail: karolinebonan25@gmail.com. Orcid: https://orcid.org/0000-0003-3169-7608.

${ }_{9}^{9}$ Discente de Terapia Ocupacional. Universidade Federal do Paraná (UFPR), Curitiba, Paraná, Brasil. Email: nilsondefreitas120@ gmail.com. Orcid: https://orcid.org/0000-0003-3063-8227.

${ }^{10}$ Discente de Enfermagem. Universidade Federal do Paraná (UFPR), Curitiba, Paraná, Brasil. E-mail: hpbruna1998@gmail.com. Orcid: https://orcid.org/0000-0003-4454-3579.
} 


\section{RESUMO}

O Programa de Educação pelo Trabalho para a Saúde (PET-Saúde) se instaurou desde 2008 enquanto política nacional indutora de transformações na formação em saúde. Em sua proposta organizacional, o PET-Saúde prevê o pacto colaborativo e interprofissional entre instituições de ensino e da saúde para a construção de ações integradas, desenvolvidas por grupos tutoriais. Desta maneira, estudantes, docentes e profissionais dos serviços de saúde se unem em projetos e ações colaborativas realizadas em campo, de maneira a transformar processos de trabalho e qualificar tanto o ensino como a prática em saúde. O presente artigo descreve o relato das experiências do PET-Saúde, desenvolvido pela Universidade Federal do Paraná em parceria com os municípios de Curitiba e Piraquara, tendo como base a descrição das ações, situações e reflexões vivenciadas pelo grupo tutorial "Educação popular, mobilização e controle social na Saúde", no período pandêmico de março de 2020 a março de 2021. O artigo, dessa forma, relata inicialmente a estruturação do PET-Saúde UFPR, apresentando ações desenvolvidas pelo coletivo voltadas a minimizar o impacto da Covid-19 em pessoas em situação de rua. Destaca-se, ainda, a elaboração de materiais de educação popular sobre a Covid-19 e ações de mobilização popular para garantir condições básicas de subsistência e cuidado contra a doença. Para além, ao relato se integram as ações desenvolvidas para o fortalecimento do controle social e da defesa dos direitos humanos. Ressalta-se a importância de políticas públicas que promovam o estabelecimento de projetos interinstitucionais, impactando de maneira positiva a formação e a especialização das práticas em saúde.

Palavras-chave: Saúde Mental. Controle Social. Educação em Saúde. Covid-19. Política Pública.

\section{ABSTRACT}

The Education through Work for Health Program (PET-Saúde) has been proposed since 2008 as a national policy that induces changes in health education. In its proposal, PET-Saúde institutes a collaborative and interprofessional pact between educational and health institutions for the construction of integrated actions, developed by tutor groups. In this way, students, teachers and health service professionals come together in collaborative projects and actions carried out in the field, in order to transform work processes and qualify both teaching and practice in health. This article reports the experiences of PET-Saúde, developed by the Federal University of Paraná in partnership with the municipalities of Curitiba and Piraquara, based on the description of the actions, situations and reflections experienced by the tutorial group "Popular education, mobilization and social control in Health", in the pandemic period from March 2020 to March 2021. The article, therefore, initially reports the structuring of PET-Saúde UFPR, presenting actions developed by the collective aimed at minimizing the impact of Covid-19 on people in street situation. Also noteworthy is the elaboration of popular education materials about Covid-19 and popular mobilization actions to guarantee basic conditions of subsistence and care against the disease. Still, the report integrates the actions developed to strengthen social control and the defense of human rights. The importance of public policies that promote the establishment of interinstitutional projects is emphasized in order to specialize both formation and health practices.

Keywords: Mental Health. Social Control. Health Education. Covid-19. Public Policy.

\section{INTRODUÇÃO}

A partir da criação do Sistema Único de Saúde (SUS), fruto de intensos movimentos populares que tomaram lugar nas décadas de 1970 e 1980, foi afirmado o compromisso da federação na garantia da saúde da população brasileira. O SUS, ainda, tomando para si uma concepção de saúde que assume o impacto de diferentes fatores sócio-ambientais como determinantes e condicionantes das condições de saúde, afirma a importância da elaboração e execução de intervenções diversas que possam transformar 
tais realidades e, com isso, impactar positivamente na saúde da população (BRASIL, 1990a).

Tal concepção de saúde, necessariamente promotora de novas e criativas práticas, exige de seus profissionais uma formação que transpõe o modelo tradicional de saúde, bastante pautado na especialização fragmentada, curativa e hospitalocêntrica (FILHO et al., 2019; LÁZARO; SATO; TEZANI, 2018; JACOBOVSKI; FERRO, 2021). Neste sentido, procurando organizar esforços para ordenar a formação de recursos humanos na área da saúde, outra das tarefas delegadas legislativamente ao SUS, inaugura-se em 2008 uma profícua parceria entre o Ministério da Saúde e o Ministério da Educação, por meio do Programa de Educação pelo Trabalho para a Saúde (PET-Saúde) (BRASIL, 2008).

O Programa PET-Saúde, enquanto política indutora de transformações, propõe aliança entre Instituições de Ensino Superior (IES) e serviços de saúde, procurando aproximar seus recursos humanos e potências institucionais para ampliar e qualificar, indissociavelmente, tanto a formação quanto as práticas de saúde. Em sua proposta, o PET-Saúde envolve estudantes de diferentes cursos de graduação, docentes de IES e profissionais que exercem suas funções em equipamentos de saúde estratégicos, promovendo: a aproximação das IES, seus docentes e estudantes, do campo de prática, e, com isso, uma maior qualificação de seus agentes pela imersão na realidade vivenciada nos equipamentos de saúde; a ampliação e qualificação da oferta de serviços dos equipamentos, aliando de maneira planejada e sistemática recursos das IES e equipamentos de saúde; a transformação de práticas e processos de trabalho na realidade dos equipamentos de saúde, qualificando a atenção em saúde e promovendo a educação permanente de seus profissionais.

Em sua nona edição (BRASIL, 2018), o PET-Saúde foi estruturado com a intenção de elaborar propostas e executar ações embasadas na prática colaborativa e interprofissional, procurando, ainda, transformar a realidade das IES por meio a indução de mudanças curriculares, alinhadas às Diretrizes Curriculares Nacionais, que promovam a interprofissionalidade.

Por meio deste edital, o governo federal aprovou 120 projetos em variados estados brasileiros. Neste contexto, a Universidade Federal do Paraná (UFPR), em parceria com os municípios de Curitiba e Piraquara, executou um dos projetos, desenvolvendo ações locorregionais integradas por meio de cinco grupos tutoriais temáticos: Promoção à Saúde; Rede de Atenção à Saúde; Vigilância em Saúde; Práticas Integrativas em Saúde e Abordagens Grupais; e Educação Popular, Mobilização e Controle Social na Saúde. 
Tal execução, contudo, foi interpelada pela atual pandemia de Covid-19, o que exigiu dos diferentes agentes envolvidos com o projeto uma repactuação de objetivos e o (re)desenho de propostas que se adequassem à nova situação imposta pela pandemia, movendo esforços, ainda, para enfrentar alguns dos diferentes impactos sociais impostos a nossa comunidade.

Neste sentido, o presente artigo tem por finalidade descrever o relato da experiência do projeto PET-Saúde/Interprofissionalidade, desenvolvido pela parceria entre UFPR e os municípios de Curitiba e Piraquara, foco dado às ações desenvolvidas no atual momento pandêmico.

\section{MÉTODO}

Para compor o relato aqui pretendido, o presente artigo descreverá as experiências, ações, situações e reflexões vivenciadas pelo grupo tutorial "Educação Popular, Mobilização e Controle Social na Saúde" do Projeto PET-Saúde/Interprofissionalidade UFPR no período de março de 2020 a março de 2021. As experiências e reflexões foram anotadas sistematicamente, compilando informações sobre as reuniões, rodas de conversa, ações e debates entre os docentes, profissionais e estudantes envolvidos com o grupo tutorial.

Enquanto enquadre para possibilitar a análise dos dados, utilizou-se a matriz proporcionada pela análise hermenêutico-dialética (CAMPOS; RODRIGUES; MORETTI-PIRES, 2012).

Inicialmente, será exposta a configuração do grupo tutorial em tela, situando o leitor a respeito de seu funcionamento organizacional e propostas. Na sequência, congregar-se-ão as experiências de maneira temática, possibilitando a composição de reflexões e a compreensão sobre as diferentes ações realizadas pelo coletivo para dar suporte à nossa comunidade frente às situações e implicações impostas pela pandemia de Covid-19.

\section{COVID-19 E A EXECUÇÃO DO PROJETO PET-SAÚDE/ INTERPROFISSIONALIDADE UFPR: ATORDOAMENTOS E POSICIONAMENTOS}


O projeto PET-Saúde/Interprofissionalidade, apresentado pela Universidade Federal do Paraná em resposta ao edital n. 10, de 23 de julho de 2018, foi elaborado de maneira a desenvolver ações locorregionais integradas nos municípios de Curitiba e Piraquara. Em sua proposta, o coletivo se organizou em cinco grupos tutoriais: Promoção à Saúde; Rede de Atenção à Saúde; Vigilância em Saúde; Práticas Integrativas em Saúde e Abordagens Grupais; e Educação Popular, Mobilização e Controle Social na Saúde.

Inicialmente, para a compreensão das propostas do Grupo Tutorial "Educação Popular, Mobilização e Controle Social na Saúde", foco deste relato de experiência, é importante uma breve situação a respeito do Controle Social.

A partir da constituição de 1988, foi firmado o compromisso social e democrático de garantir a participação da população brasileira no controle e fiscalização das ações públicas no campo da Saúde (BRASIL, 1988). Neste contexto, a lei 8.142/90 foi promulgada com o intuito de estruturar tal participação, regulamentando o controle social (BRASIL, 1990b).

O Controle Social no campo da Saúde prevê, logo, a existência de duas instâncias colegiadas, as Conferências e Conselhos de Saúde, compostas, cada qual, por $50 \%$ de representantes de usuários do SUS, 25\% de representantes de trabalhadores da saúde e $25 \%$ de representantes da gestão ou de prestadores de serviços da saúde (BRASIL, 1990b). Enquanto as Conferências de Saúde acontecem no máximo a cada quatro anos, consultando a comunidade de maneira a possibilitar sua participação na elaboração de demandas e propostas que guiem as ações e políticas públicas; os Conselhos de Saúde congregam pessoas, representantes dos segmentos de usuários, trabalhadores e gestores/prestadores de serviço, para acompanhar ininterruptamente as ações e políticas públicas desenvolvidas, fiscalizando e controlando sua execução, inclusive nos aspectos operacionais e financeiros (BRASIL, 1990b; BRASIL, 2002).

Embora tal previsão legal já tenha tomado lugar desde 1990, diferentes autores apontam problemáticas cotidianas para o exercício do controle social. As dificuldades são variadas e atravessam: a falta de conhecimento da população sobre o tema; a incipiência de formações e capacitações de conselheiros e participantes das conferências; dificuldades burocráticas e falta de apoio e estrutura para o exercício do controle social; a presença de relações de poder diversas, que impactam sobremaneira este exercício democrático; o distanciamento dos representantes de sua população representada, etc. (CORTÊS, 2009; JUNGLOS, et al., 2019; FERRO, 2014; SANTOS; FERRO, 2021). 
Neste contexto, o projeto PET-Saúde/Interprofissionalidade previu a constituição do grupo tutorial "Educação Popular, Mobilização e Controle Social na Saúde", referência dos integrantes do projeto para articular ações de mobilização popular e de fortalecimento do exercício do controle social, de maneira a potencializar a conquista de políticas públicas voltadas a responder a diferentes demandas comunitárias. Enquanto objetivos, o grupo tutorial em discussão organizou ações voltadas à construção de conhecimento, inclusive com a constituição e manutenção de grupos e assessorias a lideranças comunitárias para fortalecer o controle social, garantindo a ampliação e especialização da participação comunitária e democrática no controle e fiscalização das ações públicas.

O ano de 2020, contudo, foi marcado pelo surgimento da doença Covid-19, a qual afetou sobremaneira a população mundial, impondo a todos os países uma grande mudança de seus costumes sociais para evitar os efeitos deletérios e impactos negativos da doença. Neste contexto, as ações, ainda mais as que se ancoravam na realização de grupos e encontros diversos e, consequentemente, promoviam aglomerações, tiveram que ser reposicionadas para se adequarem às novas condições impostas pela pandemia. Ainda, os encontros de orientação e organização das ações, os quais congregavam estudantes, trabalhadores dos serviços de saúde e professores tutores e coordenadores, passaram a acontecer de maneira remota, exigindo de todos o uso de tecnologias diversas.

A partir da apropriação das formas virtuais de comunicação e promoção do trabalho, o coletivo pôde discutir as diferentes implicações advindas da pandemia para a realidade locorregional atendida pelo projeto. Neste sentido, as discussões encontraram como estrutura organizacional algumas ferramentas próprias ao Planejamento Estratégico Situacional (ARTMANN, 2000) e foi composto um quadro que reuniu ações e metas, a serem executadas pela equipe, voltadas ao enfrentamento de alguns dos impactos da pandemia em nossa comunidade.

O grupo tutorial "Educação Popular, Mobilização e Controle Social na Saúde", o qual contou com cerca de 18 integrantes no período da pandemia, divididos entre estudantes, preceptores e docentes, passou a organizar, então, encontros mensais remotos com a equipe completa para alinhamento das ações. Ainda, enquanto proposta organizacional do trabalho, os diferentes integrantes se dividiram dentre as metas elaboradas, compondo, desta forma, pequenos grupos de trabalho executivos, os quais construíram ferramentas de comunicação virtual, alinhando ações e combinando encontros de acordo com as demandas de trabalho. 
Algumas das ações realizadas serão detalhadas nos itens abaixo, apresentando reflexões e apontamentos sobre suas potencialidades e barreiras, assim como estratégias promovidas pelo grupo tutorial para fortalecer seu impacto e contornar as dificuldades vivenciadas.

\section{COVID-19 E AÇÕES DE CONSTRUÇÃO DE CONHECIMENTO E APOIO}

A primeira das dificuldades, vivenciadas amplamente em contexto nacional, foi a pluralidade de informações, muitas delas advindas de notícias falsas, sobre a Covid-19 e sobre as formas de proteção contra a doença. Neste sentido, a equipe trabalhou na produção de conteúdos sobre a doença e a utilização de máscaras e formas de proteção, procurando, ainda, divulgá-los amplamente em redes sociais construídas pelos recursos humanos vinculados ao projeto.

Paralelamente, uma população bastante afetada, tanto pela doença, como por suas condições de vida e pelas mudanças sociais vivenciadas no contexto brasileiro foram as pessoas em situação de rua. (PAULA et al., 2020). Com a circulação social da população diminuída e com o fechamento ou diminuição do funcionamento de espaços de cuidado e apoio a pessoas em situação de rua, como restaurantes populares, parques e banheiros públicos, situações bastante dificultosas foram vivenciadas por este segmento social, o qual passou a não encontrar estrutura para garantir sua higienização, alimentação e condições mínimas de subsistência.

Uma das ações articuladas pelo projeto PET-Saúde/interprofissionalidade UFPR foi a composição inicial de um e-book divulgado amplamente, com versão física e digital, estruturado a partir de especificidades próprias às pessoas em situação de rua. O material, produzido em parceria com o governo do Estado do Paraná e com a Universidade Estadual de Ponta Grossa, disponibilizou informações sobre o Covid-19, o funcionamento da doença e formas e cuidados para evitar a contaminação e sua propagação (DITTERICH et al., 2020).

A equipe discutiu também a necessidade da inserção de dados sobre instituições de controle social e de defesa dos direitos humanos. Desta forma, foram inseridas informações, inclusive com a disponibilização de contatos locorregionais, sobre o Ministério Público, Defensoria Pública, Ouvidoria, Call-Centers com informações sobre a Covid-19, Conselhos de Saúde, Associações e movimentos do terceiro setor, entre outros (DITTERICH et al., 2020). No material, ainda, foi inserido um link para um 
arquivo, composto pelo coletivo, que congrega informações sobre vários equipamentos comunitários que proporcionam suporte à população em situação de rua, com a intenção de publicizar contatos e iniciativas, fortalecendo o acesso aos serviços e a mobilização de articulações locorregionais que venham a impactar positivamente no cuidado desta população.

Ainda, por meio dos recursos humanos do projeto, foram estruturados sites institucionais e redes sociais para duas associações voltadas à defesa dos direitos da população em situação de rua de Curitiba e região. Para além de proporcionar visibilidade às ações das instituições, foram organizados espaços de denúncia nos sites, inclusive anônimas, procurando aproximar as lideranças comunitárias de sua base, fortalecendo sua representação, a defesa dos direitos humanos e o controle social.

Em contexto pandêmico já apresentado, por conta da grande necessidade vivenciada pela população em situação de rua de Curitiba para garantia de condições básicas de alimentação, instituições variadas e movimentos do terceiro setor, com destaque ao Movimento Nacional da População de Rua, Instituto Nacional de Direitos Humanos da População de Rua (INRua), Projeto Mãos Invisíveis, Itinerante resistência, Rede de Saúde Mental e Economia Solidária de Curitiba e Região Metropolitana (LIBERSOL), Associação Arnaldo Gilberti, dentre outras tantas parcerias institucionais, reuniram esforços para a produção de alimentação, distribuindo diária e gratuitamente cerca de 350 marmitas durante todo o período da pandemia. Unindo-se a esta ação, o projeto PET-Saúde/Interprofissionalidade da UFPR congregou forças com o projeto de extensão "Rede de Atenção Psicossocial (RAPS): Construindo o cuidado em liberdade e possibilidades de inclusão pelo trabalho", também vinculado à UFPR, para mobilizar e apoiar a produção da alimentação, o que possibilitou a tecitura de várias parcerias comunitárias para a doação e compra de alimentos.

A criação, previamente mencionada, de plataformas de comunicação das instituições em redes sociais e sites pelo projeto possibilitou a ampla divulgação da iniciativa, o que angariou ainda mais apoio comunitário e doações de recursos financeiros e humanos para a produção dos alimentos. A orquestração destas forças comunitárias possibilitou a conquista da parceria com uma empresa de eletrodomésticos, a qual doou à iniciativa dois freezers que vêm possibilitando estrutura para as ações e, com elas, cuidado às pessoas em situação de rua no contexto da pandemia.

Procurando dar apoio às duas associações mencionadas, uma das metas integradas entre os dois projetos PET-Saúde e RAPS foi a assessoria das instituições, no sentido de 
organizá-las burocraticamente para que possam atender às demandas do novo marco regulatório do terceiro setor (BRASIL, 2014). Para fortalecer as associações em sua atuação no controle social, na advocacia e defesa dos direitos humanos e na captação de recursos, vêm sendo organizada a inscrição burocrática das entidades: nos Conselhos Municipais de Assistência Social locorregionais; em programas voltados a proporcionar a doação de itens apreendidos na receita federal; em programas de doação de notas fiscais e de parte do imposto de renda de pessoas físicas e jurídicas; e em projetos de captação de recursos por meio de crowdfunding (MALLMANN, 2010; SANTOS; NEGRÃO; SABOYA, 2018).

O projeto PET-Saúde/Interprofissionalidade UFPR pôde unir esforços para realizar uma pesquisa documental para dar visibilidade a várias iniciativas nacionais e internacionais, públicas e/ou privadas, voltadas a dar suporte à população em situação de rua durante a pandemia de Covid-19. A pesquisa incluiu a busca de notícias, normas técnicas e documentos normativos, materiais informativos, sites e vídeos, os quais congregavam ações realizadas para dar suporte e enfrentar os impactos negativos impostos pela pandemia a esta população. No que tange aos resultados, a pesquisa coletou 07 artigos científicos, 161 notícias, 19 materiais entre recomendações e notas técnicas, portarias/decretos, protocolos de atendimento e materiais informativos como cartilhas e manuais.

A partir da pesquisa, foram levantadas experiências nacionais e internacionais que promoveram apoio quanto a questões de moradia e condições básicas de subsistência e higienização para a população em situação de rua. Neste sentido, destaca-se: a utilização de ginásios, escolas públicas, centros recreativos e clubes em desuso para acomodação e oferta de banho e alimentação; convênios com hotéis, inclusive com a distribuição de vouchers para pessoas em situação de rua; a abertura de centros de acolhimento para população em situação de rua com Covid-19; a instalação de torneiras e estrutura de pias acionadas pelo joelho, com dispensador de sabão, em pontos estratégicos da cidade; a gratuidade de restaurantes populares para pessoas em situação de rua; a utilização de estacionamentos vazios para alojar pessoas em situação de rua em barracas, com distanciamento apropriado, acesso à água potável, tomadas e energia elétrica, áreas de refeição, trailer para banho e estrutura para dispensa de lixos; a oferta por equipamentos públicos e pelo terceiro setor de kits de higienização; disponibilização na cidade de Los Angeles - Estados Unidos - de trailers estatais para garantir condições de moradia e isolamento social adequados; oferta por diferentes movimentos comunitários de 
atendimento médico, banhos, lanches/refeições, roupas e outros serviços; distribuição de excedentes de uniformes pelo correio para a produção de máscaras; instalação em pontos de apoio para pessoas em situação de rua, com estrutura para banho, kits alimentação e higiene; instalação de lavatórios perto de espaços habitados por pessoas em situação de rua.

Enquanto estrutura de funcionamento de vários espaços e iniciativas, foram ressaltadas como medidas: o mapeamento de territórios em maior vulnerabilidade e identificação da população em situação de rua; estratégias de orientação da população em situação de rua por profissionais, distribuição de panfletos e utilização de carros de som para prover orientações sobre a doença e formas de proteção; banhos iniciais para recepção das pessoas em situação de rua em unidades de acolhimento, de maneira a ampliar a higienização e evitar formas de contágio; manutenção de janelas abertas, higienização constante, distanciamento de camas e suspensão de visitas e eventos em centros de acolhimento.

Diferentes materiais encontrados ressaltaram: iniciativas de testagem de Covid19 e avaliação realizada por equipes médicas na rua, contando com transporte para abrigos e/ou para quartos de hotel reservados para quarentena; mobilização do corpo de bombeiros para montagem de clínica para prover testes gratuitos e rápidos, alinhados com encaminhamentos para locais para quarentena, quando necessário; a importância de comitês intersetoriais para discutir e propor ações voltadas ao apoio para pessoas em situação de rua.

\section{PET-SAÚDE/INTERPROFISSIONALIDADE, CONTROLE SOCIAL E CONSTRUÇÃO DE CONHECIMENTO PARA A DEMOCRACIA}

Compreendendo a importância de ações consistentes e sistemáticas para a construção de conhecimento sobre o Controle Social e sobre formas de participação democrática no estado brasileiro, ainda mais necessárias em épocas em que este funcionamento vem sendo bastante agredido e questionado, o Projeto PETSaúde/Interprofissionalidade pôde congregar suas forças e produzir diferentes materiais.

A partir da edificação de plataformas virtuais de comunicação com a população, o grupo tutorial "Educação Popular, Mobilização e Controle Social na Saúde" organizou seus recursos humanos para produzir conteúdos em formato de postagens e vídeos, voltados a divulgar amplamente o funcionamento e estrutura do Controle Social na Saúde, 
inclusive apresentando algumas formas operacionais de participação popular nos Conselhos e Conferências de Saúde.

Procurando enfrentar as dificuldades apontadas por Ferro, Ditterich e Burnagui (2019), Côrtes (2009), Junglos et al. (2019) e amplamente por autores que estudam o fenômeno do controle social, no que tange à falta de conhecimento da população sobre o tema, o coletivo produziu dois e-books com distribuição gratuita e ampla divulgação (FERRO et al., 2021a, 2021b). O primeiro pôde ressaltar a estrutura do Controle Social e a configuração dos Conselhos e Conferências de Saúde, apresentando, ainda, possibilidades de instrumentalização da população para sua participação democrática no controle das ações e políticas públicas no campo da Saúde.

A partir da clareza sobre a necessidade da população, e em especial de conselheiros e lideranças envolvidos com o Controle Social, possuir uma grande organicidade com variadas instituições de controle e defesa dos direitos humanos para garantir o enfrentamento das problemáticas do campo da Saúde, também advogada por Oliveira (2014) e Ferro, Burnagui e Ditterich (2019), o e-book em tela foi composto (FERRO et al., 2021b). No material, foram inseridos dados sobre o funcionamento de instituições, inclusive com contatos locorregionais, como a Ouvidoria, o Ministério Público, o Tribunal de Contas, as Comissões Legislativas, a Ouvidoria, a Defensoria Pública, dentre outras.

O segundo e-book, por sua vez, pôde apresentar e elaborar uma problemática vivenciada intensamente no primeiro ano de execução do projeto, no qual, fora do período de pandemia, seus recursos humanos puderam participar presencialmente de várias Conferências de Saúde.

$\mathrm{Na}$ experiência, estudantes, profissionais e docentes puderam vivenciar fenomenicamente a intensa dificuldade da organização de diferentes segmentos para a participação nas Conferências de Saúde. A partir desta constatação, foi produzido um ebook, cujo foco foi dado às Conferências de Saúde. O material foi configurado para apresentar seu funcionamento organizacional, assim como algumas sugestões e possibilidades práticas para a elaboração e defesa conjunta de propostas voltadas a guiar as ações e políticas públicas (FERRO et al., 2021a).

Para além, ações realizadas por meio de eventos de extensão em formatos remotos, e que se desdobraram em vídeos com disponibilização gratuita e online, puderam compor cena, possibilitando a discussão ampla com a população sobre o controle social. Tais materiais, enquanto estratégias de difusão e construção de conhecimento, foram 
estruturados contando também com links para outros materiais produzidos que pudessem aprofundar os conhecimentos abordados e instrumentalizar a população sobre formas de encaminhamento de denúncias e/ou sugestões para guiar as ações públicas, fortalecendo, desta forma, a prática democrática do controle social e a defesa dos direitos humanos.

\section{CONCLUSÃO}

A partir do objetivo de executar ações voltadas a fortalecer a educação popular, a mobilização e o controle social, o grupo tutorial, vinculado ao projeto PETSaúde/Interprofissionalidade da Universidade Federal do Paraná e realizado em parceria com os municípios de Curitiba e Piraquara, pôde desenvolver diferentes ações, imprescindíveis para dar suporte à população brasileira em momentos de pandemia.

Neste sentido, o PET-Saúde/Interprofissionalidade afirma sua potência enquanto estratégia promotora de aproximações institucionais e vínculos comunitários, construídos entre Universidade e variadas instituições comunitárias. Ao que compete às propostas do grupo tutorial "Educação Popular, Mobilização e Controle Social na Saúde", diferentes conquistas podem ser sublinhadas: o investimento na mobilização e estabelecimento de parcerias comunitárias para dar suporte às necessidades básicas de cuidado da população em situação de rua em época de pandemia, inclusive com a sistematização de diferentes experiências; o apoio e assessoria a instituições voltadas à defesa dos direitos humanos e ao exercício do controle social; a produção necessária, ainda mais em tempos de pandemia e de diferentes agressões aos direitos humanos, de plataformas virtuais e de materiais acadêmicos voltados ao fortalecimento do controle social, a instrumentalizar o encaminhamento de denúncias e a aproximar lideranças comunitárias e população.

Ressalta-se, por fim, a importância da estruturação de políticas públicas que promovam parcerias comunitárias diversas, tais como o Programa de Educação pelo Trabalho para a Saúde (PET-Saúde), e que reduzam a distância entre Universidade e comunidade, para que esta instituição ateste sua presença, cotidiana e sistemática, no enfrentamento, coletivo e colaborativo, das diferentes problemáticas sociais.

\section{REFERÊNCIAS}


ARTMANN, E. O planejamento estratégico situacional no nível local: um instrumento a favor da visão multissetorial. In: Oficina social $\mathbf{n}^{\mathbf{0}}$ 3: desenvolvimento social. Rio de Janeiro: COPPE/UFRJ, 2000. p. 1-25.

BRASIL. [Constituição (1988)]. Constituição da República Federativa do Brasil. Brasília, DF: Senado Federal, 1988.

Guia do conselheiro: curso de capacitação de conselheiros estaduais e municipais de saúde. Brasília: Ministério da Saúde, 2002.

Lei $n^{0} 8.080$ de 19 de Setembro de 1990. Dispõe sobre as condições para a promoção, proteção e recuperação da saúde, a organização e o funcionamento dos serviços correspondentes e dá outras providências. Diário Oficial da União, Brasília, DF, n. 182, 20 set. 1990a. Seção 1, p. 1.

Lei n⿳ 8.142 de 28 de dezembro de 1990. Dispõe sobre a participação da comunidade na gestão do Sistema Único de Saúde - SUS e sobre as transferências intergovernamentais de recursos financeiros na área da saúde e dá outras providências. Diário Oficial da União, Brasília, DF, 31 dez. 1990b.

Lei $\mathbf{n}^{\circ}$ 13.019, de 31 de julho de 2014. Estabelece o regime jurídico das parcerias entre a administração pública e as organizações da sociedade civil, em regime de mútua cooperação, para a consecução de finalidades de interesse público e recíproco, mediante a execução de atividades ou de projetos previamente estabelecidos em planos de trabalho inseridos em termos de colaboração, em termos de fomento ou em acordos de cooperação; define diretrizes para a política de fomento, de colaboração e de cooperação com organizações da sociedade civil; e altera as Leis $n^{\circ}$ s 8.429, de 2 de junho de 1992, e 9.790, de 23 de março de 1999. Diário Oficial da União, Brasília, DF, n. 146, 01 ago. 2014. Seção 1, p. 1.

. Ministério da Saúde. Edital $\mathbf{n}^{\circ} 10,23$ de julho 2018 seleção para o Programa de Educação pelo Trabalho para a Saúde PETSaúde/Interprofissionalidade - 2018/2019. Diário Oficial da União, Brasília, DF, n. 141, 24 jul. 2018. Seção 3, p. 78.

Portaria Interministerial $\mathrm{n}^{\circ} \mathbf{1 . 8 0 2}$, de 26 de agosto de 2008. Institui o Programa de Educação pelo Trabalho para a Saúde - PET - Saúde. Diário Oficial da União, Brasília, DF, 27 ago. 2008.

CAMPOS, D. A. de.; RODRIGUES. J.; MORETTI-PIRES, R. O. Pesquisa em Saúde Coletiva como instrumento de transformação social: uma proposta fundamentada no pensamento hermenêutico-dialético. Saúde \& Transformação social, Florianópolis, v. 3, n. 4, p. 14-24, 2012.

CÔRTES, S. V. Participação e Saúde no Brasil. Rio de Janeiro: Editora Fiocruz, 2009.

DITTERICH, R. G. et al. Covid-19: orientações à população em situação de rua.

Ponta Grossa: UEPG/PROEX, 2020. E-book. Disponível em: 
https://www2.uepg.br/proex/wp-content/uploads/sites/8/2020/06/Pessoas-emsitua\%C3\%A7\%C3\%A3o-de-rua-1.pdf. Acesso em: 08 abr. 2021.

FERRO, L. F. et al. Controle social e conferências de saúde: o que são e como podemos participar para construir a saúde que precisamos. Curitiba: Editora da UFPR, 2021a. E-book. Disponível em: encurtador.com.br/eJYZ5. Acesso em: 08 abr. 2021.

Controle social e participação popular. Curitiba: Editora da UFPR, 2021b. E-book. Disponível em: encurtador.com.br/bnw24. Acesso em: 08 abr. 2020.

FERRO, L. F. Controle Social e Saúde Mental: barreiras e estratégias para o exercício do controle social. 2014. 264f. Tese (Doutorado em Psicologia Social) Instituto de Psicologia, Universidade de São Paulo, São Paulo, 2014.

FERRO, L. F.; BURNAGUI, J. G.; DITTERICH, R. G. Controle Social e Saúde Mental: estratégias para a construção coletiva do SUS. In: CREMASCO, M. V. F.; MENZ, D. M. (Org.). Formação em Políticas sobre Drogas. Curitiba: Editora UFPR, 2019. p. 253-282.

FILHO, J. R. F. et al. Educação Interprofissional nas políticas de reorientação da formação profissional em saúde no Brasil. Saúde em Debate, Rio de Janeiro, v. 43, n. 1, p. 86-96, ago. 2019. http://dx.doi.org/10.1590/0103-11042019s107.

JACOBOVSKI, R.; FERRO, L. F. Permanent education in Health and Active Learning methodologies: a systematic integrative review. Research, Society and Development, [S.1.], v. 10, n. 3, p. e39910313391, 2021. http://dx.doi.org/10.33448/rsdv10i3.13391. Disponível em: encurtador.com.br/lsSW2. Acesso em: 23 mar. 2021.

JUNGLOS, C. et al. Motivações, importância, desafios e perspectivas do controle social em saúde. Cogitare Enfermagem, [S.1.], v. 24, não p. 2019. http://dx.doi.org/10.5380/ce.v24i0.66874.

LÁZARO, A. C.; SATO, M. A. V.; TEZANI, T. C. R. Metodologias ativas no ensino superior: o papel do docente no ensino presencial. 2018. Não paginado. Disponível em: encurtador.com.br/nBE79. Acesso em: 08 mar. 2021.

MALLMANN, F. Incentivos fiscais relacionados a doação: uma alternativa para a prática da responsabilidade social. Monografia de graduação (Bacharelado em Ciências Contábeis) - Universidade Federal do Rio Grande do Sul, Porto Alegre, 2010.

OLIVEIRA, V. de C. Comunicação, informação e participação popular nos Conselhos de Saúde. Saúde e Sociedade, São Paulo, v. 13, n. 2, p. 56-69, ago. 2004. https://doi.org/10.1590/S0104-12902004000200006.

PAULA, H. C. et al . Sem isolamento: etnografia de pessoas em situação de rua na pandemia de Covid-19. Revista Brasileira de Enfermagem, Brasília, v. 73, não p. 2020.

SANTOS, G. W. dos.; FERRO, L. F. Controle social e saúde mental: estudo sobre barreiras e possibilidades para a participação social. Revista Extensão em Foco, Curitiba, n. 22, p. 84-103, jan./jul. 2021. 
SANTOS, Y. da C. dos.; NEGRÃO, K. R. M.; SABOYA, S. M. P. Estratégias para captação de recursos no terceiro setor: um estudo multicaso aplicado na APAE Belém e APAE Barcarena. Revista de Administração e Contabilidade da Faculdade Estácio do Pará, Belém, v. 5, n. 10, p. 175-213, dez. 2018.

Recebido em: 13 de abril de 2021.

Aceito em: 20 de abril de 2021. 